\title{
Digitalization in Medicine: It Is Our Chance and Responsibility Now to Shape the Digital Future of Breast Cancer Management
}

\author{
Nadia Harbeck \\ Brustzentrum, Frauenklinik und CCCLMU der Universität München (LMU), Munich, Germany
}

Our life, public and private, is becoming digitalized whether we like it or not, and so are medicine and patient management. Probably no other change in the way we practice medicine is currently met with so many emotions. Personal preference, ethics, and concerns regarding privacy protection impact on our opinion of the new advances in digital technology.

Unfortunately, there still is an international discrepancy regarding digitalization in medicine due to resources, priorities, and legislation that substantially impacts on implementation of digital advances in our own clinical environment. In Germany, digitalization in education, industry, and society is currently being promoted by the government. Nevertheless, in medicine, the speed of digitalization differs greatly between different hospitals as well as between hospitals and private practices. In the hospital environment, interdisciplinary and inter-professional use of digital technologies in patient care is not clearly regulated; responsibilities between nurses and physicians have not yet been properly defined, which hampers the application of digital tools in the daily clinical work flow. Moreover, research projects regarding digitalization suffer from the fact that they sometimes reach beyond current legal boundaries and this lack of precedence explains why IRBs tend to be rather conservative with such proposals. This leads to sometimes unnecessary hurdles, which in turn demotivate researchers and slow down interesting and forward-looking projects.

In medicine, advancing digitalization for the benefit of patients and caregivers requires a problem-solving mentality rather than prospectively imposed detailed legislation and regulatory barriers that discourage and frustrate engaged health care workers and their patients both in clinical routine and in research.

In oncology, the groundbreaking work by Basch et al. [1] and Denis et al. [2] has demonstrated that electronic capture of PROs is superior to routine clinical care with regard to patient satisfaction, quality of life as well as patient outcome. This approach also seems to be cost-effective for the respective medical system. These results strongly suggest that we need to implement new digital technologies and thus modernize patient management.

Cancer patients are quite willing to embrace new digital ways of communication if it helps them to speed up diagnostic and therapeutic procedures, and ensures specialized expert opinion or close communication with their own treatment team. Data protection tends to be commonly confused with data security. This misunderstanding then results in an overload of bureaucracy in a clinical environment where time is already a scarce commodity. Why not empower patients to decide themselves which digital advances they want to utilize instead of even banning modern communication, such as emails containing clinical reports, from the hospital care setting. Investing in and ensuring data security is mandatory in promoting digitalization. Nevertheless, patients should be able to decide themselves which level of data protection is appropriate for them as they already do this in their everyday life anyway.

Digitalization in medicine is certainly the future. It holds great promise for education of health care professionals, diagnosis, and treatment as well as everyday patient management as outlined by the three wonderful review articles in this issue of Breast Care. These articles

\section{KARGER}

(c) 2019 S. Karger AG, Basel
Univ.-Prof. Dr. med. Nadia Harbeck

Brustzentrum, Frauenklinik

Universität München (LMU)

Marchioninistrasse 15, DE-81377 Munich (Germany)

E-Mail Nadia.Harbeck@med.uni-muenchen.de 
allow a glimpse into the promise of digitalization in breast cancer care but obviously do not cover its whole potential which also extends to surgery, pathology, and several other aspects of multidisciplinary breast cancer management.

Digital data collection improves speed, quality assurance, and transparency, but also comes with responsibilities. We need to ensure data security as well as carefully implement these new technologies and validate new diagnostic or decision algorithms that impact directly on patient care. Regarding modern forms of communication between patients and their treatment team, we also need to make sure that patient safety is ensured and that responsibilities are clearly defined. Unless a 24/7 communication channel is properly staffed, apps such as CANKADO can only help patients to prepare for their next doctor's visit but not replace seeing the doctor in person in case of an emergency.

Digitalization in medicine needs to continue to be a topic of academic research such as exemplified by the work by Basch et al. [1] as well as the ongoing PRECYCLE study (NCT 03220178). It must be driven by the needs of patients and health care professionals and not by those of industry. At present, we can still decide whether we are just going to implement what is being forced on us or whether we would like to embrace digitalization, direct and shape it for the benefit of patients and their clinical care.

References

1 Basch E, Dueck AC, Rogak LJ, Mitchell SA, Minasian LM, Denicoff AM, et al. Feasibility of Implementing the Patient-Reported Outcomes Version of the Common Terminology Criteria for Adverse Events in a Multicenter Trial: NCCTG N1048. J Clin Oncol. 2018 Sep; 36(31):JCO2018788620.

2 Denis F, Basch E, Septans AL, Bennouna J, Urban T, Dueck AC, et al. Two-Year Survival Comparing Web-Based Symptom Monitoring vs Routine Surveillance Following Treatment for Lung Cancer. JAMA. 2019 Jan; 321(3):306-7. 